\title{
AUTHOR REPLY: The nuances of GRADE
}

\author{
Trustin Domes, MD, MEd'; Borna Tadayon Najafabadi, MD, MPH²; Matthew Roberts, MD, MEdi3; \\ Jeffrey Campbell, $M D^{4}$; Ryan Flannigan, $M D^{5}$; Phil Bach, $M D^{6}$; Premal Patel, $M D^{7} ;$ Gavin Langille, $M D^{8}$; \\ Yonah Krakowsky, $M D^{9}$; Philippe D. Violette, $M D, M S c^{2,10}$
}

\begin{abstract}
'Division of Urology, Department of Surgery, University of Saskatchewan, Saskatoon, SK, Canada; ${ }^{2}$ Department of Health Research Methods, Evidence and Impact, McMaster University, Hamilton, ON, Canada; ${ }^{3}$ Division of Urology, Department of Surgery, University of Ottawa, Ottawa, ON, Canada; ${ }^{4}$ Division of Urology, Department of Surgery, Western University, London, ON, Canada; ${ }^{5}$ Department of Urological Sciences, University of British Columbia, Vancouver, BC, Canada; 'Division of Urology, Department of Surgery, University of Alberta, Edmonton, AB, Canada; ${ }^{7}$ Division of Urology, Department of Surgery, University of Manitoba, Winnipeg, MB, Canada; ${ }^{8}$ Saint John Regional Hospital, Saint John, NB, Canada; ' ${ }^{2}$ ivision of Urology, Women's College Hospital \& Sinai Health System, Department of Surgery, University of Toronto, Toronto, ON, Canada; ${ }^{10}$ Department of Surgery, McMaster University, Hamilton, ON, Canada
\end{abstract}

Cite as: Domes T, Najafabadi BT, Roberts M, et al. Author reply: The nuances of GRADE. Can Urol Assoc J 2021;15(12):426-8. http://dx.doi.org/10.5489/cuaj.7679

W e would like to thank the members of the British Society for Sexual Medicine (BSSM) for their feedback and well-intentioned comments regarding the recently published Canadian Urological Association (CUA) erectile dysfunction (ED) guideline. ${ }^{1}$ We appreciate having this platform to respond to their concerns and clarify our position.

We sincerely believe that the primary divergence between us is a matter of understanding the nuances of the Grading of Recommendations Assessment, Development, and Evaluation (GRADE) approach ${ }^{2}$ and the evidence-todecision (EtD) framework ${ }^{3}$ by which the recommendations were formulated. The results of our analyses are available transparently in the guideline Appendix. This well-established framework considers multiple factors (importance of the question, desirable effects, undesirable effects, certainty in the evidence of effects, patient values and preferences, the balance of desirable and undesirable effects, resources required, certainty about resources required, cost effectiveness, equity, acceptability to stakeholders, and feasibility of implementing the recommendation) to formulate patient-centric and less biased recommendations based on the analysis of high-quality evidence. The CUA is making a concerted effort to develop guidelines using a strong methodological framework, such as GRADE. Guidelines not using such frameworks risk the loss of transparency in their reasoning behind recommendations and are prone to overstating the strength of recommendations. It is misleading to state a position strongly because one feels strongly. A strong recommendation must be supported in consideration of the quality of available evidence and other important factors for clinical decision-making, which are encompassed in the EtD framework.

In the EtD framework, a conditional recommendation does not imply that "everyone should do" the course of action recommended. Importantly, this approach and a con- ditional recommendation stress that the clinician is not making the decision for the patient but that the clinician's role is to help the patient regarding the best course of action for them through a shared decision-making process. The best course of action is the one that most aligns with patient's values and preferences when considering the relevant tradeoffs. This approach is, in our opinion, the ultimate way to practice patient-centered and evidence-informed medicine.

In their letter, BSSM members note that we do not recommend daily tadalafil as a treatment option for patients with $E D$. This statement is a misinterpretation of the recommendation and is not accurate. We made a conditional recommendation against the general preferential use of daily tadalafil rather than on-demand tadalafil (or any other on-demand phosphodiesterase type 5 inhibitor [PDE5i]); however, both treatment options are acceptable, and the patient-specific choice will be based on multiple factors.

Many of the comments made by members of the BSSM in favor of daily tadalafil are, in fact, accounted for in the guideline when it states that certain patient-centered factors may influence what dosing regimen the patient ultimately decides to pursue. To illustrate, if a patient with ED has a high desire for sexual spontaneity, then daily tadalafil may be a better option for them. Additionally, if a patient with ED has comorbid symptomatic benign prostatic hyperplasia, then of course daily tadalafil may be a good option for them. Conversely, factors such as cost, desire not to take a daily medication, and frequency of intercourse, may lead a patient to consider on-demand dosing based on their values and preferences. Preference for daily vs. on-demand dosing lies with the patient based on their values and preferences instead of clinicians preferentially prescribing daily-dose regimens for their patient with ED.

We absolutely agree with and support the involvement of the patient's partner in discussions regarding sexual health and ED assessment and management. We applaud investigators that include this important perspective in their work and hope more researchers capture the partner's perspective in future studies. The study by Conaglen and colleagues 
demonstrated that female partners preferred daily or ondemand tadalafil over sildenafil. ${ }^{4} \mathrm{~A}$ followup study of the same cohort of patients in the "real-world" setting with two years of followup interestingly found that long-term adherence to PDE5is was only $71 \%$, and despite this, the vast majority of couples continued to be sexually active and satisfied without treatment. ${ }^{5}$ As well, many couples required less medication than on trial, citing less need for them and the high cost as the two main factors for decreased use. This data speaks to the natural history of couples presenting with sexual concerns over time and that numerous factors play a role in medication preference, dosing frequency, and continued use.

The guideline committee used the International Index of Erectile Function (IIEF)-EF score to measure improvement in erectile function when comparing studies, which is the most robust validated measure used in trials assessing ED outcomes. ${ }^{6}$ When analyzing the randomized controlled trials (RCTs) that compared daily vs. on-demand tadalafil, the followup period of 8-12 weeks was chosen because the vast majority of studies reported data with this followup period. A systematic review that investigated longer followup periods, from 24-36 weeks, demonstrated very similar results as our meta-analysis (pooled mean difference in IIEF-EF of 1.24 [95\% confidence interval $0.03-2.44) ;{ }^{7}$ however, one of the four included studies actually only had 12 weeks of followup given its crossover design. ${ }^{8}$ We included three of the four studies from the Zhou systematic review in our metaanalysis, using 12-week followup data for direct comparison between RCTs to decrease heterogeneity between studies. In our literature search, we were unable to find RCTs or studies to support the claim made by members of the BSSM that longer followup demonstrates increasing superiority for daily tadalafil vs. on-demand regimens.

In the guideline, we reiterate that for treatment-refractory patients, clinicians should review dosing, technique, side effects, and consider re-treatment, which could include changing from an on-demand to a daily dose PDE5i regimen. In the letter from members of the BSSM, daily tadalafil is quoted to effectively salvage $50 \%$ of men failing on-demand PDE5is. In our literature search, very little high-quality evidence to support this claim was found, especially with a $50 \%$ salvage rate. The only study that quoted a salvage rate close to $50 \%$ is an open-label study of 101 men determined to have failed on-demand tadalafil and $37 \%$ more men taking tadalafil $10 \mathrm{mg}$ daily compared to tadalafil $20 \mathrm{mg}$ ondemand responded positively to the SEP3 question (Did your erection last long enough for you to have successful intercourse?). ${ }^{9}$ This study also reports that $41 \%$ of patients taking tadalafil $10 \mathrm{mg}$ daily had an IIEF-EF score equal to or better than 26 compared to zero patients taking tadalafil on-demand. Apart from its small size, there are numerous methodological flaws and biases in this study that limit its applicability to the general ED population (method used to determine tadalafil failure, very narrow inclusion criteria, no measure of frequency of on-demand use, no mention of why failure occurred, no mention of education provided to on-demand treatment failures, failure to report raw IIEF scores in each treatment group, and the sole author has a disclosed conflict of interest to the company that manufactures tadalafil), and practically, tadalafil $10 \mathrm{mg}$ is not approved for daily use.

In contrast, a study by Hatzimouratidis and colleagues determined that proper patient education regarding ondemand PDE5i use was more effective in salvaging nonresponding patients than daily dosing regimens. ${ }^{10}$ Discussing a daily dose regimen for a patient that is non-responsive to on-demand PDE5-inhibitors after ensuring proper use through patient education is reasonable (as is discussing a change for a patient not responding to daily dose to ondemand); however, given the similar clinical efficacy of the dosing regimens in patients with $\mathrm{ED}$, we don't feel giving the patient the choice threatens their potential treatment success or future relationship with their partner.

As an argument in favor of daily dosing, members of the BSSM highlighted potential benefits of long-term daily tadalafil beyond its effect on sexual function. These additional effects, apart from improvements in voiding function, are seen in longitudinal studies that are hypothesis-generating and don't form the bedrock of evidence in guideline creation, especially when RCT data in multiple trials directly addressing the clinical question exist. We look forward to future studies evaluating the protective role of PDE5-inhibitors to help bring further clarity to these fascinating associations.

We concede that this guideline has limitations and unanswered questions, as our colleagues from the BSSM allude to. We distinctly address these at the end of the guideline document and specifically state that the guideline is focused on patients with ED as a single presenting symptom, albeit we acknowledge many patients will have other urological conditions that may impact their ED management. We also state that "the recommendations in this guideline need to be contextualized based on the patient's history and presenting symptoms and conditions that may be influencing sexual function in its entirety." This statement is not an excuse for not digging deeper into the literature; it is a function of high-quality evidence not existing to adequately address these clinical circumstances and others in the field of ED. We hope more evidence emerges to address these gaps so guideline recommendations can reflect unbiased, balanced, and evidence-informed decisions to guide clinicians treating $E D$ in the future. 


\section{References}

1. Domes T, Najafabadi BT, Roberts M, et al. Canadian Urological Association guideline: Erectile dysfunction. Can Urol Assoc J 2021;15:303-15. https://doi.org/http://dx.doi.org/10.5489/cuaj.7572

2. Guyatt GH, Oxman AD, Vist GE, et al. GRADE: An emerging consensus on rating quality of evidence and strength of recommendations. BMJ 2008;336:924-6. https://doi.org/10.1136/bmi.39489.470347. AD

3. Alonso-Coello P, Schunemann HJ, Moberg J, et al. GRADE Evidence to Decision (EtD) frameworks: A systematic and transparent approach to making well-informed healthcare choices. 1: Introduction. BMJ 2016;353:i2016. htrps://doi.org/10.1136/bmi.i2016

4. Conaglen HM, Conaglen JV. Investigating women's preference for sildenafil or tadalafil use by their partners with erectile dysfunction: the partners' preference study. I Sex Med 2008;5:1198-207. hitps://doi.org/10.1111/i.1743-6109.2008.00774.x

5. Conaglen HM, Conaglen JV. Couples' reasons for adherence to, or discontinuation of, PDE type 5 inhibitors for men with erectile dysfunction at 12 to 24 -month followup after a 6 -month free trial. J Sex Med 2012;9:857-65. https://doi.org/10.1111/j.1743-6109.2011.02625.x
6. Rosen RC, Cappelleri JC, Gendrano N, 3rd. The International Index of Erectile Function (IIEF): A stateof-the-science review. Int J Impot Res 2002;14:226-44. https://doi.org/10.1038/si.ijir.3900857

7. Zhou Z, Chen $\mathrm{H}$, Wu J, et al. Meta-analysis of the long-erm efficacy and tolerance of tadalafil daily compared with tadalafil on-demand in treating men with erectile dysfunction. Sex Med 2019;7:282-91. https://doi.org/10.1016/i.esxm.2019.06.006

8. McMahon C. Comparison of efficacy, safety, and tolerability of on-demand tadalafil and daily dosed tadalafil for the treatment of erectile dysfunction. J Sex Med 2005;2:415-25; discussion 25-7. htrps://doi.org/10.1111/i.1743-6109.2005.20360.x

9. McMahon C. Efficacy and safety of daily tadalafil in men with erectile dysfunction previously unresponsive to on-demand tadalafil. J Sex Med 2004;1:292-300. https://doi.org/10.1111/i.1743-6109.04042.x

10. Hatzimouratidis K, Moysidis K, Bekos $A$, et al. Treatment strategy for "non-responders" to tadalafil and vardenafil: A real-life study. Eur Urol 2006;50:126-32; discussion 32-3. https://doi.org/10.1016/i. eururo.2006.02.060 